Xue Mei, Xiang Yong, Wang Jian, Zhang Yushan, Yao Yuehua and Wang Jianlong*

\title{
The crystal structure of 3-chloro-1-hydrazino-2,4, 6-trinitrobenzene, $\mathrm{C}_{6} \mathrm{H}_{4} \mathrm{ClN}_{5} \mathrm{O}_{6}$
}

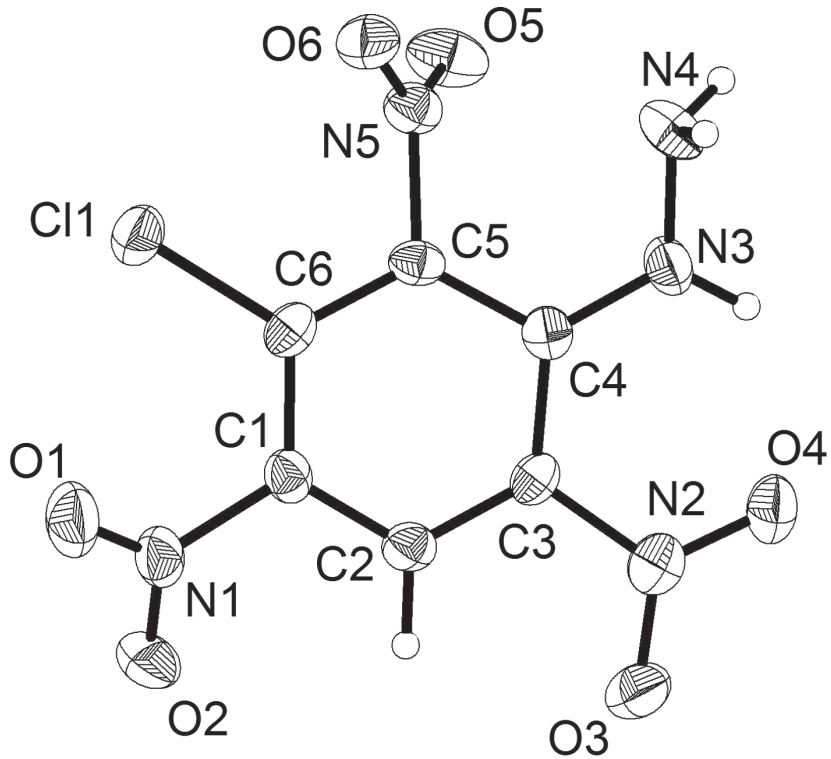

https://doi.org/10.1515/ncrs-2019-0647

Received September 2, 2019; accepted October 17, 2019; available online November 8, 2019

\section{Abstract}

$\mathrm{C}_{6} \mathrm{H}_{4} \mathrm{ClN}_{5} \mathrm{O}_{6}$, monoclinic, $P 2_{1} / n$ (no. 14), $a=10.2139$ (13) $\AA$, $b=5.6835(6) \AA, \quad c=17.597(2) \AA, \quad \beta=106.484(4)^{\circ}$, $V=979.6(2) \AA^{3}, \quad Z=4, \quad R_{\mathrm{gt}}(F)=0.0497, \quad w R_{\text {ref }}\left(F^{2}\right)=0.1138$, $T=173 \mathrm{~K}$.

\section{CCDC no.: 1959969}

The molecular structure is shown in the figure. Table 1 contains crystallographic data and Table 2 contains the list of the atoms including atomic coordinates and displacement parameters.

*Corresponding author: Wang Jianlong, School of Chemical Engineering and Technology, North University of China, Taiyuan 030051, Shanxi Province, P.R. China, e-mail: 619379961@qq.com Xue Mei, Zhang Yushan and Yao Yuehua: School of Chemical Engineering and Technology, North University of China, Taiyuan 030051, Shanxi Province, P.R. China. https://orcid.org/0000-00022210-5124 (X. Mei)

Xiang Yong: Sichuan North Hongguang Special Chemical Co., Ltd. Yibin 644104, Sichuan Province, P.R. China

Wang Jian: No. 113, Tianjin Road, Jianxi District, Luoyang 471003, Henan Province, P.R. China

๖ Open Access. ๑ 2019 Xue Mei et al., published by De Gruyter. (cc)BY License.
Table 1: Data collection and handling.

\begin{tabular}{ll}
\hline Crystal: & Yellow block \\
Size: & $0.08 \times 0.06 \times 0.04 \mathrm{~mm}$ \\
Wavelength: & Mo $K \alpha$ radiation $(0.71073 \AA)$ \\
$\mu:$ & $0.43 \mathrm{~mm}^{-1}$ \\
Diffractometer, scan mode: & Bruker APEX-II, $\varphi$ and $\omega$ \\
$\theta_{\text {max }}$, completeness: & $26.4^{\circ}, 99 \%$ \\
$N\left(h k l_{\text {measured }}, N(h k l)_{\text {unique }}, R_{\text {int }}:\right.$ & $7003,1974,0.070$ \\
Criterion for $I_{\text {obs }}, N\left(h k l l_{\mathrm{gt}}:\right.$ & $I_{\text {obs }}>2 \sigma\left(I_{\text {obs }}\right), 1268$ \\
$N(\text { param })_{\text {refined }}:$ & 171 \\
Programs: & Bruker [1], Olex2 [2], SHELX [3, 4]
\end{tabular}

Table 2: Fractional atomic coordinates and isotropic or equivalent isotropic displacement parameters $\left(\AA^{2}\right)$.

\begin{tabular}{lrrrr}
\hline Atom & $\boldsymbol{x}$ & $\boldsymbol{y}$ & $\boldsymbol{z}$ & $\boldsymbol{U}_{\text {iso }}{ }^{*} \boldsymbol{U}_{\text {eq }}$ \\
\hline Cl1 & $0.61771(8)$ & $0.79091(15)$ & $0.50526(5)$ & $0.0355(3)$ \\
O1 & $0.9015(2)$ & $0.6515(5)$ & $0.55432(14)$ & $0.0455(7)$ \\
O2 & $0.9138(2)$ & $0.2714(5)$ & $0.56408(14)$ & $0.0475(7)$ \\
O3 & $0.7745(2)$ & $-0.1102(4)$ & $0.31743(15)$ & $0.0432(7)$ \\
O4 & $0.5901(2)$ & $-0.0182(4)$ & $0.22709(13)$ & $0.0365(6)$ \\
O5 & $0.3144(2)$ & $0.5900(4)$ & $0.35412(14)$ & $0.0421(7)$ \\
O6 & $0.4257(2)$ & $0.9114(4)$ & $0.34942(14)$ & $0.0358(6)$ \\
N1 & $0.8632(3)$ & $0.4537(5)$ & $0.53113(16)$ & $0.0335(7)$ \\
N2 & $0.6758(3)$ & $0.0201(5)$ & $0.29058(17)$ & $0.0308(7)$ \\
N3 & $0.4650(3)$ & $0.3801(5)$ & $0.23775(15)$ & $0.0309(7)$ \\
H3 & 0.459213 & 0.254406 & 0.207780 & $0.037^{*}$ \\
N4 & $0.3823(3)$ & $0.5769(5)$ & $0.20935(18)$ & $0.0349(7)$ \\
N5 & $0.4178(3)$ & $0.6978(5)$ & $0.35429(16)$ & $0.0291(6)$ \\
C1 & $0.7524(3)$ & $0.4303(5)$ & $0.45836(18)$ & $0.0253(7)$ \\
C2 & $0.7578(3)$ & $0.2478(5)$ & $0.40926(18)$ & $0.0263(7)$ \\
H2 & 0.829735 & 0.136294 & 0.424932 & $0.032^{*}$ \\
C3 & $0.6611(3)$ & $0.2220(5)$ & $0.33753(18)$ & $0.0232(7)$ \\
C4 & $0.5519(3)$ & $0.3844(5)$ & $0.30993(18)$ & $0.0237(7)$ \\
C5 & $0.5443(3)$ & $0.5575(5)$ & $0.36709(18)$ & $0.0238(7)$ \\
C6 & $0.6411(3)$ & $0.5870(5)$ & $0.43848(18)$ & $0.0250(7)$ \\
H4A & $0.303(3)$ & $0.529(5)$ & $0.1891(19)$ & $0.027(9)^{*}$ \\
H4B & $0.417(4)$ & $0.658(7)$ & $0.174(2)$ & $0.063(13)^{*}$ \\
\hline & & & &
\end{tabular}

\section{Source of material}

2,4-Dichloro-1,3,5-trinitrobenzene was reacted with an appropriate amount of hydrazine hydrate at room temperature for half an hour. After filtering and drying, the crude product 
was obtained and purified by column chromatography to give the title compound. Yellow crystals were obtained by slowly evaporating an ethyl acetate solution at room temperature.

\section{Experimental details}

C2- and N3-bound $\mathrm{H} 2$ and $\mathrm{H} 6$ atoms were placed geometrically and refined as riding with $U_{\text {iso }}(\mathrm{H})=1.2 U_{\text {eq }}(\mathrm{C}, \mathrm{N})$. The N4-bound $\mathrm{H}$-atoms were located from difference Fourier maps and their positions and isotropic temperature factors freely refined.

\section{Comment}

Nitrophenyl hydrazine derivatives have broad applications in the fields of medicinal and materials chemistry; they can be used as test reagents for carbonyl groups and as intermediates for the synthesis of pharmaceuticals [5-9].

In the crystal structure of the title compound all bond lengths and angles are within normal ranges and in good agreement with those found for the structure of 2,4,6-trinitrophenylhydrazine [10] and 1,3-dichloro-2,4,6trinitrobenzene [11]. The structure of the title compound (cf. the figure) has one benzene ring, and three nitro groups, one hydrazine group and one chlorine as substituents. The dihedral angles between the nitro groups and the benzene ring are $36.89(17), 4.24(17)$ and $65.73(17)^{\circ}$ for the nitro groups at N1, N2 and N5, respectively. The dihedral angle between the hydrazine group and the benzene ring is $15.8(17)^{\circ}$ (N4, N3, C4). The hydrazine group donates a total of four hydrogen bonds, including an intramolecular one: $\mathrm{N} 3-\mathrm{H} 3 \cdots 04[\mathrm{~N} 3 \cdots \mathrm{O} 4=2.632(44) \AA$ with angle at $\mathrm{H} 3=127], \mathrm{N} 3-\mathrm{H} 3 \cdots \mathrm{O} 2^{\mathrm{i}}\left[\mathrm{N} 3 \cdots \mathrm{O} 2^{\mathrm{i}}=3.074(4) \AA\right.$ with angle at $\mathrm{H} 3=129$ for (i) $-1 / 2+x, 1 / 2-y,-1 / 2+z]$; $\mathrm{N} 4-\mathrm{H} 4 \mathrm{a} \cdots \mathrm{O}^{\mathrm{ii}} \quad\left[\mathrm{N} 4 \cdots \mathrm{O} 6^{\mathrm{ii}}=3.163(4) \AA\right.$ with angle at $\mathrm{H} 4 \mathrm{a}=171(3)^{\circ}$ for (ii) $\left.1 / 2-x,-1 / 2+y, 1 / 2-z\right]$ and $\mathrm{N} 4-\mathrm{H} 4 \mathrm{~b} \cdots \mathrm{O} 1^{\mathrm{iii}}\left[\mathrm{N} 4 \cdots \mathrm{O} 1^{\mathrm{iii}}=3.189(4) \AA\right.$ with angle at $\mathrm{H} 4 \mathrm{~b}=154(3)^{\circ}$ for (iii) $\left.-1 / 2+x, 3 / 2-y,-1 / 2+z\right]$.
Acknowledgements: We are grateful for the support of the Shanghai Institute of Materia Medica, Chinese Academy of Sciences.

\section{References}

1. Bruker. APEX3, SAINT and SADABS. Bruker AXS Inc., Madison, WI, USA (2016).

2. Dolomanov, O. V.; Bourhis, L. J.; Gildea, R. J.; Howard, J. A. K.; Puschmann, H.: OLEX2: a complete structure solution, refinement and analysis program. J. Appl. Crystallogr. 42 (2009) 339-341.

3. Sheldrick, G. M.: SHELXT - integrated space-group and crystalstructure determination. Acta Crystallogr. A71 (2015) 3-8.

4. Sheldrick, G. M.: Crystal structure refinement with SHELXL. Acta Crystallogr. C71 (2015) 3-8.

5. Allen, C. F. H.: The identification of carbonyl compounds by use of 2,4-dinitrophenylhydrazine. J. Am. Chem. Soc. 52 (1930) 2955-2959.

6. Lewis, S. A.; Connatser, R. M.; Olarte, M. V.; Keiser, J. R.: Determining aromatic and aliphatic carboxylic acids in biomassderived oil samples using 2,4-dinitrophenylhydrazine and liquid chromatography-electrospray injection-mass spectrometry/mass spectrometry. Biomass Bioenergy 108 (2018) 198-206.

7. Jun, H.; Rehan, H.; Lim, M. D.; Kieran, A.; Juncong, Y.; Karen, L.; Christoph, H. B.: Isotope-labeling derivatization with 3-nitrophenylhydrazine for $\mathrm{LC} /$ multiple-reaction monitoringmass-spectrometry-based quantitation of carnitines in dried blood spots. Anal. Chim. Acta 1037 (2018) 177-187.

8. Atabaki, F.; Keshavarz, M. H.; Bastam, N. N.: Synthesis and investigation of the new derivatives of poly (epichlorohydrin) containing energetic groups. Propell. Explos. Pyrot. 43 (2018) 83-89.

9. Liu, G.; Hu, Y. D.; Wang, Y. H.: Crystal structure of (E)-1-[1(3-chloro-4-fluorophenyl)ethylidene]-2-(2,4-dinitrophenyl) hydrazine, $\mathrm{C}_{28} \mathrm{H}_{20} \mathrm{Cl}_{2} \mathrm{~F}_{2} \mathrm{~N}_{8} \mathrm{O}_{8}$. Z. Kristallogr. NCS 232 (2017) 627-629.

10. Flippen-Anderson, J. L.; Dudis, D. S.: Structure of 2,4,6trinitrophenylhydrazine. Acta Crystallogr. C45 (1989) 1107-1109.

11. Holden, J. R.; Dickinson, C.: The crystal structure of 1,3dichloro-2,4,6-trinitrobenzene. J. Phys. Chem. 71 (1967) $1129-1131$. 